\title{
Dual phononic and photonic band gaps in a periodic array of pillars deposited on a thin plate
}

\author{
Y. El Hassouani, ${ }^{1}$ C. Li, ${ }^{1}$ Y. Pennec,,${ }^{1} *$ E. H. El Boudouti,${ }^{1,2}$ H. Larabi, ${ }^{1}$ A. Akjouj, ${ }^{1}$ O. Bou Matar, ${ }^{1}$ V. Laude,${ }^{3}$ \\ N. Papanikolaou, ${ }^{4}$ A. Martinez, ${ }^{5}$ and B. Djafari Rouhani ${ }^{1}$ \\ ${ }^{1}$ Institut d'Electronique, de Microélectronique et de Nanotechnologie, Université de Lille 1, 59655 Villeneuve d'Ascq, France \\ ${ }^{2}$ Laboratoire de Dynamique et d'Optique des Matériaux, Faculté des Sciences, Université Mohamed I, 60000 Oujda, Morocco \\ ${ }^{3}$ Institute FEMTO-ST, ENSMM, Université de Franche Comté, Besançon, France \\ ${ }^{4}$ Institute of Microelectronic, NCSR, Athens, Greece \\ ${ }^{5}$ Nanophotonics Technology Center, Universidad Politécnica de Valencia, Valencia, Spain
}

(Received 31 July 2010; revised manuscript received 7 September 2010; published 4 October 2010)

\begin{abstract}
We study theoretically the simultaneous existence of phononic and photonic band gaps in a periodic array of silicon pillars deposited on a homogeneous thin silica plate. Several lattices, namely, square, triangular, and honeycomb are investigated for a wide range of geometrical parameters. We discuss the most suitable cases for dual phononic-photonic band gaps, especially in comparison to the more conventional structures constituted by a periodic array of holes in a membrane.
\end{abstract}

DOI: 10.1103/PhysRevB.82.155405

PACS number(s): 43.40.+s, 42.70.Qs, 42.79.Jq

\section{INTRODUCTION}

Phononic crystals are a class of materials that exhibit periodic variations in their density and elastic properties. ${ }^{1-3}$ Such crystals modify the propagation of acoustic waves and prohibit the propagation of sound for frequencies within the band gap. They have enabled exciting new ways to control sound, in particular, in the field of wave guiding and filtering, ${ }^{4}$ using point and linear defects introduced in the crystal, as well as in the field of sound isolation. ${ }^{5}$ Recently, an issue of interest is based on the study of phononic crystal slabs for potential applications as platforms for integrated technological circuits. Most of the studies have been devoted to periodic arrays of holes drilled in a homogeneous plate $e^{6-10}$ whereas only a few recent works have been dealt with pillar structures deposited on a plate. ${ }^{11-14}$ In the latter structure, only the square-lattice geometry has been investigated where we have shown the importance and range of geometrical parameters to display absolute band gaps.

In the photonic counterpart, the medium is made up with a periodically modulation of the refractive index between their constituents also producing band gaps in which the propagation of electromagnetic waves is forbidden. ${ }^{15}$ The existence of photonic band gaps for guided modes in periodic crystal slabs offers new possibility to control the light in integrated photonic devices. Two types of photonic crystal slabs, namely, lattice of holes ${ }^{16,17}$ and lattice of pillars, ${ }^{18,19}$ have been considered. However, the case of pillars deposited on a finite thickness low-index layer, which is the only technically feasible, has not been yet reported to our knowledge.

The simultaneous existence of photonic and phononic band gaps and the confined phonon-photon interaction has been investigated in one-dimensional multilayer structures. ${ }^{20}$ In infinite two-dimensional (2D) structures, relatively few works have been devoted to simultaneous control of phonons and photons. Maldovan and Thomas ${ }^{21,22}$ have shown theoretically that phononic and photonic band gaps can be obtained in 2D square or hexagonal lattice crystals made up of air holes in a silicon matrix. Sadat-Saleh et al. ${ }^{23}$ have demonstrated the possibility to open phononic and photonic band gaps in complex arrays like multiple cylinders per unit cell in lithium-niobate 2D structures. Experimental evidence of such a band-gap phenomenon has been reported in threedimensional phononic and photonic crystal of amorphous silica spheres. ${ }^{24}$ Some recent papers are also dealing with the optomechanical crystal slabs that sustain both the optical and mechanical excitations. ${ }^{25-27}$ In two very recent papers, we and another group ${ }^{26,27}$ demonstrated the existence of dual phononic and photonic band gaps in 2.5D crystal plates composed of arrays of void cylindrical holes in silicon slabs with a finite thickness. It has been shown that dual complete band gaps can be obtained in honeycomb and boron-nitride lattices, although in a narrow range of geometrical parameters only. However, there are several possibilities in square, honeycomb, and boron-nitride lattices to display a complete phononic gap together with a photonic gap of odd or even symmetry with respect to the plane symmetry of the membrane. The triangular lattice is not very suitable for exhibiting a complete phononic gap except at very high filling fraction. Also, in all the suitable cases, a relatively high filling factor has been demonstrated as a necessity.

The main goal of this paper is to give a comprehensive study about the existence of dual phononic-photonic band gaps in the type of structure constituted by a periodic array of pillars deposited on a layer of finite thickness. As mentioned above, in the phononic side, the study of this structure has been done only in the square-lattice geometry. In this paper, we extend this investigation to other lattices, such as triangular and honeycomb and show new unexpected possibilities. On the other hand, we study, for each geometry of the lattice, the range of parameters for which this structure can display absolute photonic band gaps for guided modes parallel to the slab.

We concentrate our calculations on a structure where the pillars and the supporting plate are, respectively, made of silicon and silica $\left(\mathrm{SiO}_{2}\right)$. Besides the technological interest on silicon structures, the selection of the materials is motivated by the following. The possibility of opening phononic gaps is not much affected by the choice of the materials although the frequency and width of the gaps are material dependent. In photonic, Johnson et al. ${ }^{16}$ have studied the 

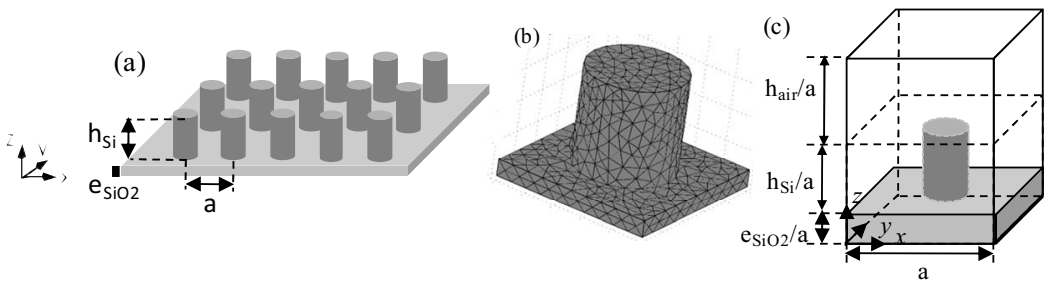

FIG. 1. (a) Schematic view of the periodic crystal made up of cylindrical $\mathrm{Si}$ pillars on a $\mathrm{SiO}_{2}$ plate. Representation of the unit cell used for: (b) the FE (phononic) and (c) the PWE (photonic) calculations.

band structure of a periodic array of silicon rods in air and shown the existence of a gap for odd symmetric modes only (magnetic field in plane). However, in this work, the height of the rods is taken equal to two times the lattice parameter and the result displays similarity with the case of an infinite 2D structure where only a TM gap can be obtained. ${ }^{16,21} \mathrm{We}$ have noticed that decreasing the height of the rods to values lower than the lattice parameter allows the opening of a complete photonic gap for the guided modes. Therefore, one can expect that the low refractive index of $\mathrm{SiO}_{2}$ may enable us to keep such a property even in presence of the thin plate.

We investigate both the phononic and photonic band structures in three types of lattices, namely, square, triangular, and honeycomb and for a wide range of the geometrical parameters. In general, the phononic and photonic band structures are, respectively, calculated by finite-element (FE) and plane-wave expansion (PWE) methods. However, finite difference time domain (FDTD) method has also been used to check the correctness and convergence of the results.

Section II describes the geometries considered in this paper as well as the methods of calculation. Sections III and IV contain the trends of the band gaps as a function of the lattice and the geometrical parameters for phononics and photonics, respectively. Section V presents some selected illustrations of the band structures for the most appropriate geometries exhibiting dual band gaps. Conclusions are given in Sec. VI.

\section{GEOMETRY AND METHOD OF CALCULATION}

Figure 1(a) represents the general schematic view of the periodical structure of cylindrical silicon pillars deposited on a thin $\mathrm{SiO}_{2}$ plate. The elastic constants and mass densities of the materials are given in Table I. The $z$ axis is chosen to be perpendicular to the plate and parallel to the cylinder axis. By considering the lattice period $a$ as the unit of length, there are several geometrical parameters involved in the problem, namely, the height $h_{\mathrm{Si}}$ of the pillars, the thickness $e_{\mathrm{SiO}_{2}}$ of the slab and the filling fraction $f$.

TABLE I. Physical characteristics of the used materials: $\rho$ is the density, $C_{11}, C_{12}$, and $C_{44}$ are the three elastic moduli, and $n$ is the refractive index.

\begin{tabular}{lcc}
\hline \hline Constant & Silicon $(\mathrm{Si})$ & Silica $\left(\mathrm{SiO}_{2}\right)$ \\
\hline$\rho\left(\mathrm{kg} / \mathrm{m}^{3}\right)$ & 2331 & 2275 \\
$C_{11}\left(\mathrm{~N} / \mathrm{m}^{2}\right)$ & $16.57 \times 10^{10}$ & $7.50 \times 10^{10}$ \\
$C_{12}\left(\mathrm{~N} / \mathrm{m}^{2}\right)$ & $6.39 \times 10^{10}$ & $2.25 \times 10^{10}$ \\
$C_{44}\left(\mathrm{~N} / \mathrm{m}^{2}\right)$ & $7.962 \times 10^{10}$ & $3.0 \times 10^{10}$ \\
$n$ & 3.5 & 1.5 \\
\hline \hline
\end{tabular}

The phononic band structures are calculated by using the FE method with the COMSOL MULTIPHYSICS finite-element software. Only the solid materials are meshed [Fig. 1(b)] since elastic waves obviously cannot propagate in vacuum. Periodic boundary conditions, using the Bloch-Floquet equations, are applied at each side of the plate, assuming an infinite and periodic structure in the $(x, y)$ plane.

On the photonic side, the calculations of the dispersion curves are performed by using the developed PWE code with periodic conditions applied on each boundary of the unit cell [see Fig. 1(c)]. In this case, the thickness of the air slab separating neighboring photonic crystals in the $z$ direction has been chosen such that to decouple them. Let us also mention that in the slab geometry, the photonic gaps have to be searched only below the light cone in vacuum. The air thickness has been chosen equal to $h_{\text {air }} / a=4.0$ to ensure the stability of the whole branches under the light cone and the calculations have been performed with a number of plane waves equal to 2499. Calculations and convergences have also been checked using the FDTD with a good agreement.

In both phononic and photonic cases, the wave vector is chosen along the high-symmetry axis of the first Brillouin zone and the eigenfrequencies are obtained by solving the eigenvalue equation. In all the band structures presented in the paper, the frequencies are given in the dimensionless unit $\Omega=\omega a / 2 \pi c$, where $c$ is the velocity of light in vacuum for electromagnetic waves and the transverse velocity of sound in silicon for elastic waves.

As illustrated in Fig. 2(a), we have investigated several lattices, namely, square, triangular, and honeycomb where the corresponding Brillouin zones are represented in Fig. 2(b). The filling fractions of the cylindrical pillars are, respectively, given by $f=\frac{\pi r^{2}}{a^{2}}, f=\frac{2 \pi r^{2}}{a^{2} \sqrt{3}}$, and $f=\frac{4 \pi r^{2}}{a^{2} \sqrt{3}}$ for the (a)

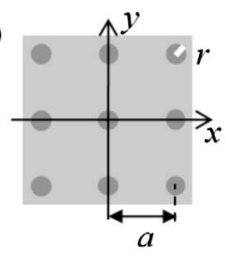

(b)

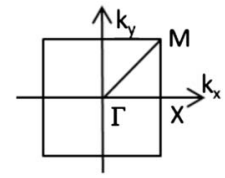

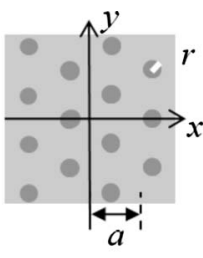
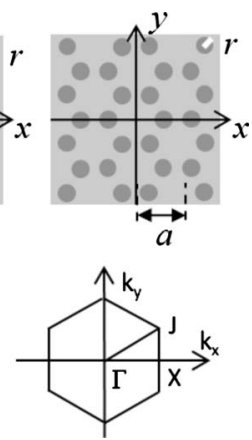

FIG. 2. (a) Square, triangular, and honeycomb arrangements of the lattice crystal made up of silicon cylinders deposited onto a $\mathrm{SiO}_{2}$ homogeneous substrate. (b) Representation of the Brillouin zone for the square (left) and for the triangular and honeycomb arrays (right). 

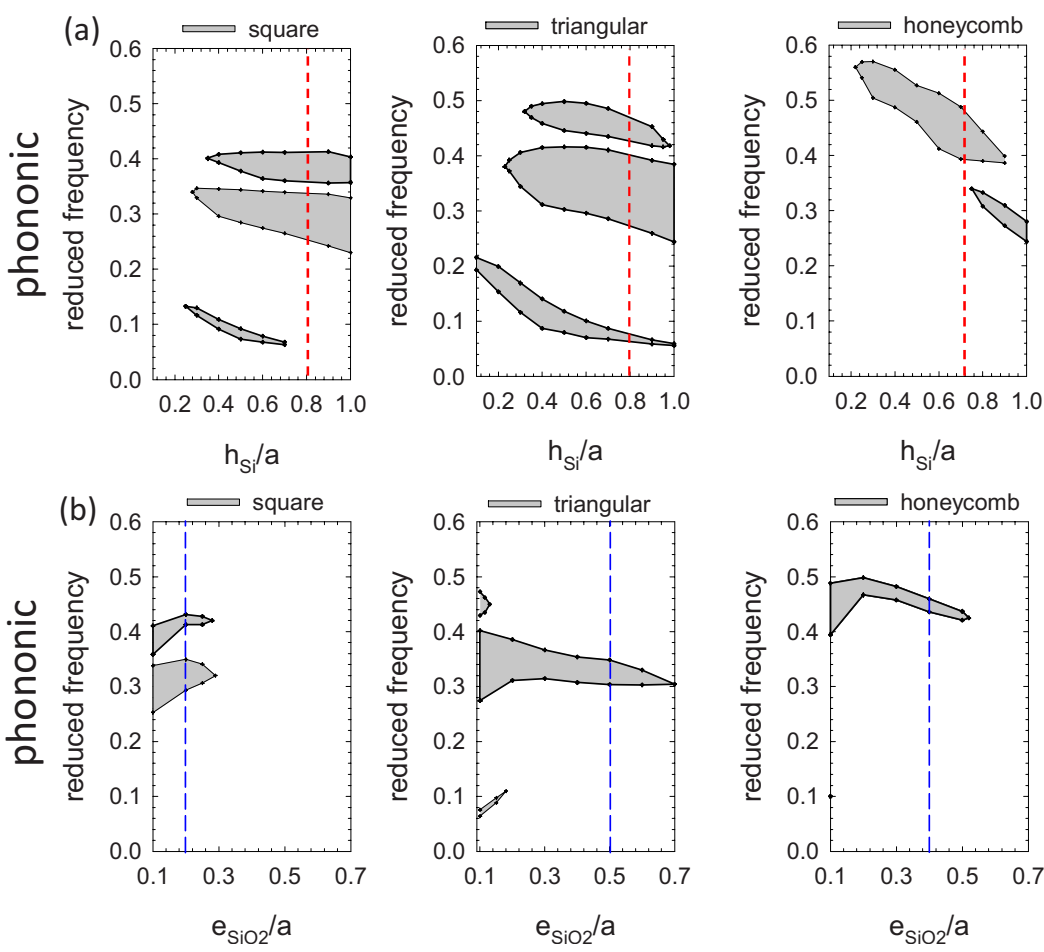

FIG. 3. (Color online) Evolution for the square, triangular, and honeycomb arrays of the phononic band gaps: (a) as a function of the height of the pillars $h_{\mathrm{Si}} / a$, for $e_{\mathrm{SiO}_{2}} / a=0.1$ and (b) as a function of the thickness of the plate $e_{\mathrm{SiO}_{2}} / a$, for $h_{\mathrm{Si}} / a=0.8$ (square and triangular) or $h_{\mathrm{Si}} / a=0.7$ (honeycomb). The filling factors of the pillars are kept at moderate values of $f=0.4$ (square), $f=0.5 \quad$ (triangular), and $f=0.3$ (honeycomb). square, triangular, and honeycomb array, where $r$ is the radius of the cylinder.

In a previous paper, ${ }^{11}$ we have demonstrated the existence of absolute phononic band gaps in a square lattice of steel cylindrical pillars on a silicon plate. It has been shown that such structure can provide acoustic band gaps when the geometrical parameters, in particular, the thickness $e$ of the homogeneous plate and the height $h$ of the pillars, are chosen appropriately; more especially $e$ should not exceed a small fraction of the period $a$. Moreover, these gaps persist for various combinations of the materials constituting the plate and the pillars. In photonic, most of the papers in the literature have been dealt with pillars of finite thickness embedded in air. We propose here a geometry where the silicon pillars are deposited on a thin plate which, contrary to the case of isolated silicon pillars, allows also the propagation of the acoustic waves via the plate. As mentioned above, the photonic gaps have to be searched only below the light cone in vacuum. Then, the choice of the material constituting the plate has to be made in such a way as to avoid too many branches under the light cone which is unfavorable for the opening of band gaps. This explains the choice of a low refractive index material such as $\mathrm{SiO}_{2}$ which, in addition, is a basic material in silicon technology.

The purpose of our study is then to find dual phononic and photonic band gaps in the three most basic lattices. The choice of the filling factors has been done considering the behavior of the photonic absolute band gaps for a thin plate $\left(e_{\mathrm{SiO}_{2}} / a=0.1\right)$. It appears from the calculations (not shown here) that a decrease in the filling factor shifts the photonic band gaps of the guided modes to the high frequencies, toward the bottom of the light cone over a small domain of the Brillouin zone, which is not useful for practical applications. On the other hand, high filling factor introduces new branches in the photonic dispersion curves that close the band gap. Therefore, contrary to the case of hollow cylinders in a Si membrane ${ }^{26,27}$ where a relatively high filling fraction is required to obtain the dual band gaps, here the filling factor can be limited to a moderate range which is interesting in view of the technological realization of the structures. We use the values of $f=0.4, f=0.5$, and $f=0.3$, respectively, for the square, triangular, and honeycomb lattices, corresponding to the reduced radii values of $r / a=0.357, r / a=0.371$, and $r / a=0.203$ for the pillars. We present in the following sections the evolution of the band gaps as a function of the most relevant parameters of the structure, namely, the thickness of the plate $e_{\mathrm{SiO}_{2}}$ and the height of the pillars $h_{\mathrm{Si}}$.

\section{PHONONIC BAND GAPS}

Figure 3(a) displays the map of the phononic band gaps for the three lattices as a function of the normalized height $h_{\mathrm{Si}} / a$ of the pillars, assuming a constant and relatively small value of the thickness of the plate $e_{\mathrm{SiO}_{2}} / a=0.1$ (as discussed in the previous section). In all three cases, one can observe the opening of gaps (gray areas) as far as the height of the pillars exceeds $0.3 a$. In the frequency range of the Bragg diffraction, around $[0.3,0.5]$, we note the existence of two band gaps in all configurations. In the square and triangular lattices, these band gaps fall around nearly constant frequencies of $\Omega=0.35$ and $\Omega=0.45$, independently of the height of the pillars. These gaps are separated by a narrow pass band. The latter is essentially constituted by nearly flat branches associated to modes which are mainly localized at the corners of the unit cell inside the thin plate without too much penetration into the pillars (let us notice that such situation of a narrow pass band separating two band gaps has already been found in several early calculations on 2D phononic crystals $^{28}$ ). When increasing the thickness of the plate [see 

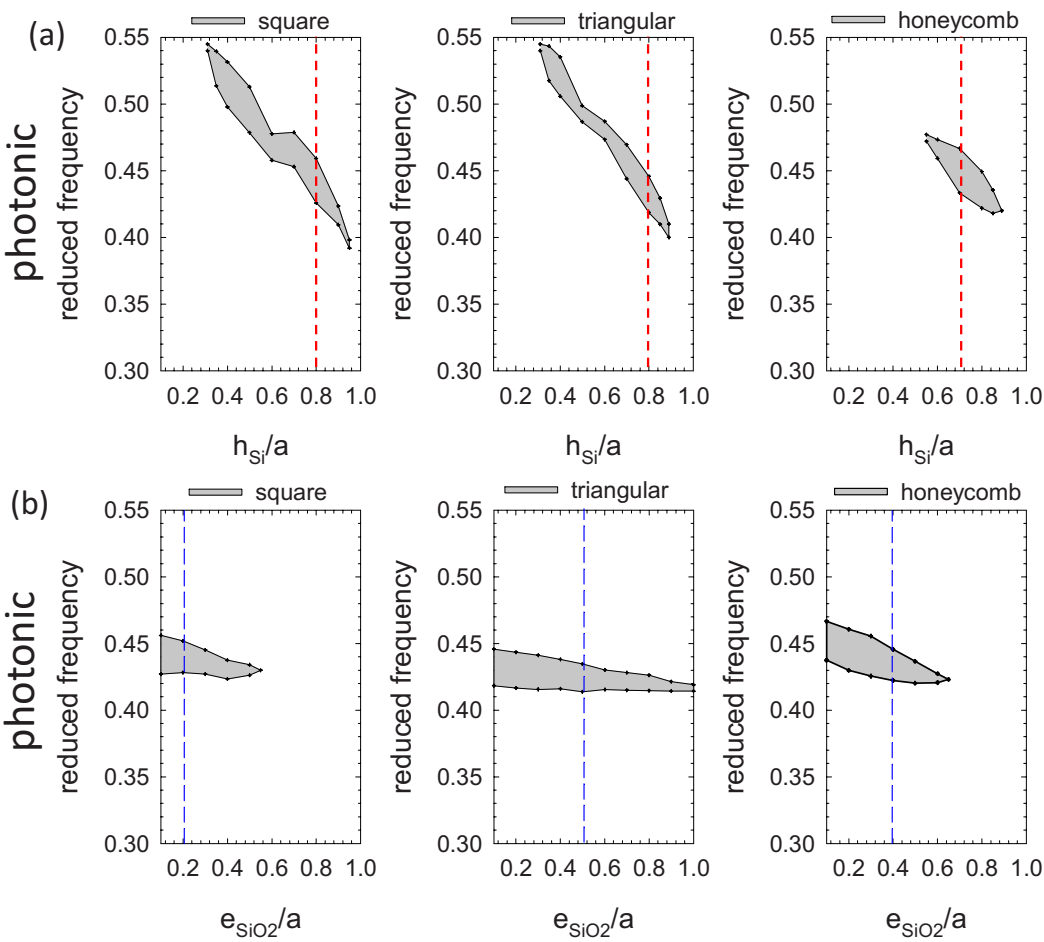

FIG. 4. (Color online) Evolution for the square, triangular, and honeycomb arrays of the photonic band gap: (a) as a function of the height of the pillars $h_{\mathrm{Si}} / a$, for $e_{\mathrm{SiO}_{2}} / a=0.1$ and (b) as a function of the thickness of the plate $e_{\mathrm{SiO}_{2}} / a$, for $h_{\mathrm{Si}} / a=0.8$ (square and triangular) or $h_{\mathrm{Si}} / a=0.7$ (honeycomb). The filling factor of the pillars is chosen to be $f=0.4$ (square), $f=0.5$ (triangular), and $f=0.3$ (honeycomb). also Fig. 3(b) and discussion below), the flat branches broaden and simultaneously higher branches move downward and, as a consequence, the higher gap closes. However, the lower gap is more robust in the triangular lattice and remains open for much higher thicknesses of the plate. In addition, for the square and triangular lattices, a narrow gap can also exist at lower frequencies $(\Omega<0.2)$, much below the Bragg regime, where the wavelength in all constituting materials is at least ten times larger than the period of the lattice. The origin and existence condition of this gap, which presents some similarity with the behavior in locally resonant sonic material, ${ }^{5}$ was discussed in detail in Ref. 11 for the square-lattice geometry. In particular, it was shown that the existence of this gap requires a very thin plate $(e / a$ $\approx 0.1$ ) and also the height of the pillars should be a moderate fraction of $a$ as can be seen in Fig. 3(a).

In Fig. 3(b) we present the variation in the gaps as a function of the thickness of the plate, for a fixed value of the height of the pillars $\left[h_{\mathrm{Si}} / a=0.8\right.$ for the square and triangular arrays and $h_{\mathrm{Si}} / a=0.7$ for the honeycomb lattice as indicated by the red vertical dashed lines in Fig. 3(a)]. In the squarelattice geometry, the band gaps close quickly with the increase in the plate thickness $\left(e_{\mathrm{SiO}_{2}} / a \geq 0.25\right)$ while for the triangular lattice the Bragg gap persists over a wide range of the thickness $\left(e_{\mathrm{SiO}_{2}} / a \leq 0.7\right)$. For the honeycomb lattice, the gap also remains as far as $e_{\mathrm{SiO}_{2}} / a \leq 0.5$, although it is narrower than in the case of the triangular lattice. Overall the central frequency of the Bragg gap does not change too much when varying $e_{\mathrm{SiO}_{2}}$. On the other hand, the lowfrequency gap in both the triangular and square lattices closes quickly when increasing $e_{\mathrm{SiO}_{2}}$. In contrast to our previous study about the square-lattice geometry, ${ }^{11}$ the triangular and honeycomb lattices can exhibit a Bragg gap for much thicker plates, which is a desirable condition for the techno- logical fabrication of such samples. Moreover, the triangular lattice is the one that exhibits the largest gap over a wider range of the geometrical parameters. Let us remember ${ }^{26,27}$ that in a structure constituted by periodic holes in a Si membrane, the triangular lattice is not at all suitable to exhibit phononic band gaps.

\section{PHOTONIC BAND GAPS}

In this section, we present the trends of the photonic band gaps as a function of the geometrical parameters. Figure 4(a) presents the photonic band gap maps as a function of the height $h_{\mathrm{Si}} / a$ of the pillars, for a constant value of the thickness of the plate $e_{\mathrm{SiO}_{2}} / a=0.1$. In all investigated lattices, one can see clearly the existence of a complete photonic gap (gray areas) which, however, closes faster than in the phononic case when increasing the height of the pillars. More precisely, for $e_{\mathrm{SiO}_{2}} / a=0.1$, the gap exists when the height of the pillars $h_{\mathrm{Si}} / a$ is chosen in the range $[0.4,0.9]$ for the square and triangular arrays and in the narrower range of $[0.6,0.8]$ for the honeycomb lattice. Actually, it would be desirable to have the frequency of the photonic gap as lowest as possible, which means choosing the highest possible values of $h_{\mathrm{Si}} / a$ around $0.7-0.8$. Otherwise the gap covers only a very small area of the Brillouin zone just below the light cone and therefore becomes not very useful for practical applications (see Sec. V and Fig. 5).

In Fig. 4(b), we give the photonic band gap maps when varying the thickness of the plate from 0.1 to 1.0 with a constant value of the pillar height $\left[h_{\mathrm{Si}} / a=0.8\right.$ for the square and triangular arrays and $h_{\mathrm{Si}} / a=0.7$ for the honeycomb one 

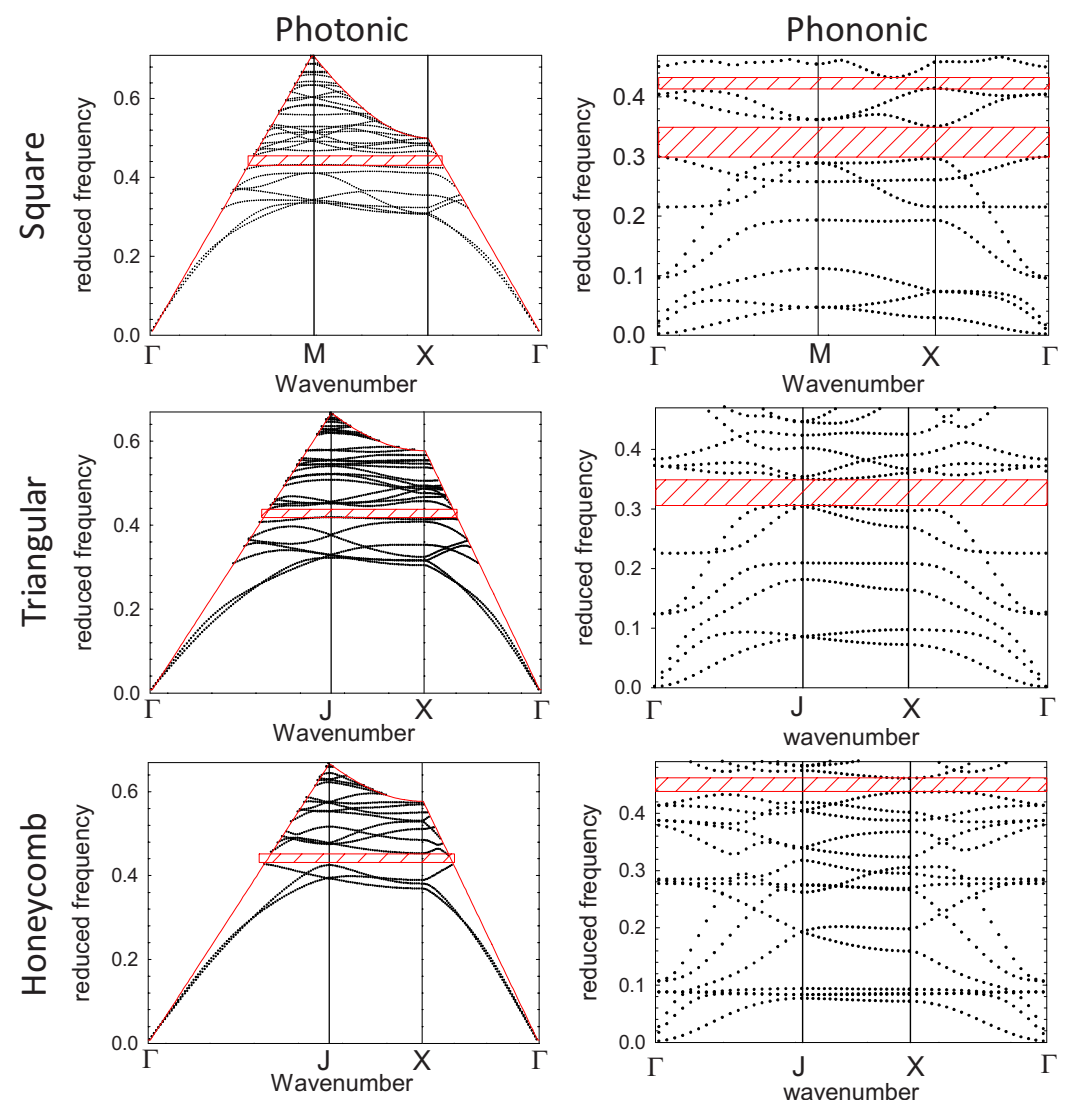

FIG. 5. (Color online) Photonic (left) and phononic (right) dispersions curves calculated for (a) the square array with the set of parameters $e_{\mathrm{SiO}_{2}} / a=0.2, h_{\mathrm{Si}} / a=0.8$, and $f=0.4$, (b) the triangular array with the set of parameters $e_{\mathrm{SiO}_{2}} / a$ $=0.5, h_{\mathrm{Si}} / a=0.8$, and $f=0.5$, and (c) the honeycomb array with the set of parameters $e_{\mathrm{SiO}_{2}} / a$ $=0.4, h_{\mathrm{Si}} / a=0.7$, and $f=0.3$. as indicated by the red vertical dashed lines in Fig. 4(a)]. In all cases, the gaps progressively close as far as the thickness of the plate increases while the central frequency of the gap changes very smoothly. Here also, the triangular lattice allows the highest value of the thickness to keep the gap open.

\section{DUAL PHOTONIC/PHONONIC BAND GAPS}

We have clearly demonstrated the existence of complete phononic and photonic band gaps for the three investigated arrays of silicon pillars on $\mathrm{S}_{2}$ plate. The conditions on the geometrical parameters to obtain dual band gaps can be expressed as follows. For the square array, the existence of a phononic gap requires a very thin plate $\left(e_{\mathrm{SiO}_{2}} / a \approx 0.1-0.2\right)$ whereas the existence of a photonic gap imposes to choose the height of the pillars as a fraction of the period $\left(h_{\mathrm{Si}} / a\right.$ $\approx 0.4-0.9$ ). In practice, to avoid the photonic gap to occur only in a very restricted domain of the Brillouin zone just below the light cone, it would be suitable to decrease its frequency and therefore to choose the highest possible value of $h_{\mathrm{Si}} / a$, around 0.8 [see Fig. 4(a)]. In comparison to the square lattice, the triangular array permits more flexibility in the choice of the parameters, especially as concerns the thickness of the plate which may become now a fraction of the period (up to $e_{\mathrm{SiO}_{2}} / a \approx 0.7$ in the above calculation with $\left.h_{\mathrm{Si}} / a=0.8\right)$. This is an interesting point to stress in view of the technological fabrication of such samples as will be shown below. Finally, the honeycomb lattice can also exhibit a dual band gap for a relatively broad range of value of $e_{\mathrm{SiO}_{2}} / a \approx 0.1-0.5$ but for a narrow range of $h_{\mathrm{Si}} / a \approx 0.6-0.9$.
Table II gives a summary of the results presented in Secs. III and IV.

Based on the above calculations, we give in Fig. 5 an illustration of both phononic and photonic dispersion curves for each of the three lattices. The geometrical parameters are those indicated by the red and blue vertical dashed lines in Figs. 3 and 4. From this figure, it can be seen that it is more suitable to find the photonic gap around or below the dimensionless frequency 0.4 , otherwise it will be restricted only to a small part of the Brillouin zone below the light cone.

Of course the actual frequencies in Figs. 3-5 scale inversely with the real dimensions of the structures. As an example, we can assume that the photonic midgap should occur at the telecommunication wavelength region around $1550 \mathrm{~nm}$. Then all the geometrical parameters become totally determined and are given in Table III. With these parameters, the photonic gap occurs in the range of 1500-1600 nm as required and the corresponding phononic gap falls in the range of a few gigahertz. As mentioned above, let us notice that, unlike the square lattice, the triangular and honeycomb lattices do not require a very thin plate.

TABLE II. Range of parameters allowing dual photonicphononic band gaps for a fixed value of the plate thickness $\left(e_{\mathrm{SiO}_{2}} / a=0.1\right)$ or a fixed value of the pillar height $h_{\mathrm{Si}} / a$.

\begin{tabular}{lccc}
\hline \hline Array & $r / a$ & $h_{\mathrm{Si}} / a$ & $e_{\mathrm{SiO}_{2}} / a$ \\
\hline Square & 0.357 & {$[0.4,0.9] 0.8$} & $0.1[0.1,0.25]$ \\
Triangular & 0.371 & {$[0.4,0.9] 0.8$} & $0.1[0.1,0.7]$ \\
Honeycomb & 0.203 & {$[0.6,0.8] 0.7$} & $0.1[0.1,0.5]$ \\
\hline \hline
\end{tabular}


TABLE III. Geometrical parameters that produce the dispersion curves of Fig. 5, assuming that the photonic midgap occurs at the telecommunication wavelength of $1550 \mathrm{~nm}$.

\begin{tabular}{lcccccc}
\hline \hline Array & $\begin{array}{c}a \\
(\mathrm{~nm})\end{array}$ & $\begin{array}{c}r \\
(\mathrm{~nm})\end{array}$ & $\begin{array}{c}h_{\mathrm{Si}} \\
(\mathrm{nm})\end{array}$ & $\begin{array}{c}e_{\mathrm{SiO}_{2}} \\
(\mathrm{~nm})\end{array}$ & $\begin{array}{c}\text { Phononic band gap } \\
(\mathrm{GHz})\end{array}$ & $\begin{array}{c}\text { Photonic band gap } \\
(\mathrm{nm})\end{array}$ \\
\hline Square & 682 & 245 & 546 & 136 & {$[2.51,3.00]$} & {$[1508,1590]$} \\
Triangular & 659 & 244 & 527 & 330 & {$[2.69,3.09]$} & {$[1511,1591]$} \\
Honeycomb & 672 & 136 & 470 & 269 & {$[3.79,4.00]$} & {$[1508,1591]$} \\
\hline \hline
\end{tabular}

\section{CONCLUSION}

We have theoretically demonstrated that a periodic array of silicon pillars deposited on a thin homogeneous $\mathrm{SiO}_{2}$ layer exhibits dual phononic/photonic complete band gaps in the three most common lattices, namely, square, triangular, and honeycomb. The geometrical parameters appear to be quite compatible with the technological fabrication facilities. In addition, the triangular lattice provides the best flexibility in the choice of the geometrical parameters which is an interesting point in view of the creation of defects such as waveguides and cavities similarly to the case of usual photonic crystals. These properties will be investigated in subsequent works.

Phononic and photonic crystal slabs hold promises for the simultaneous confinement and tailoring of sound and light waves with potential applications to acousto-optical devices and highly controllable photon-phonon interactions. The new structure studied in this paper presents an alternative with respect to the more common structure constituted by a peri- odic array of air holes in a silicon membrane. In the latter case, it has been shown ${ }^{26,27}$ that a complete photonic gap occurs only for a restricted range of the geometrical parameters in the honeycomb and boron-nitride lattices while for a wide range of parameters the phononic gap is accompanied only by a photonic gap with a given polarization (odd or even). Instead, in the case of pillars, the complete gaps can exist over a wide range of parameters. In addition, it is not required to choose a relatively high filling fraction, in contrast to the case of air holes in silicon.

\section{ACKNOWLEDGMENTS}

This work is supported in part by the European Commission Seventh Framework Programme (FP7) under the FETOpen project TAILPHOX through Grant No. 233833 and by Ministry of Higher Education and Research, Nord-Pas de Calais Regional Council and FEDER through the "Contrat de Projets Etat Region (CPER) 2007-2013." *yan.pennec@univ-lille1.fr

${ }^{1}$ M. S. Kushwaha, P. Halevi, L. Dobrzynski, and B. DjafariRouhani, Phys. Rev. Lett. 71, 2022 (1993).

${ }^{2}$ M. M. Sigalas and E. N. Economou, Solid State Commun. 86, 141 (1993).

${ }^{3}$ For a comprehensive list of references on phononic crystals, see the phononic database at http://www.univ-lehavre.fr/recherche/ lomc/phonon/PhononicDatabase1.html

${ }^{4}$ Y. Pennec, B. Djafari-Rouhani, J. O. Vasseur, H. Larabi, A. Khelif, A. Choujaa, S. Benchabane, and V. Laude, Appl. Phys. Lett. 87, 261912 (2005).

${ }^{5}$ Z. Liu, X. Zhang, Y. Mao, Y. Y. Zhu, Z. Yang, C. T. Chan, and P. Sheng, Science 289, 1734 (2000).

${ }^{6}$ J. C. Hsu and T. T. Wu, Phys. Rev. B 74, 144303 (2006).

${ }^{7}$ A. Khelif, B. Aoubiza, S. Mohammadi, A. Adibi, and V. Laude, Phys. Rev. E 74, 046610 (2006).

${ }^{8}$ J. O. Vasseur, P. A. Deymier, B. Djafari-Rouhani, and Y. Pennec, Proceeding of IMECE 2006, ASME International Mechanical Engineering Congress and Exhibition, Chicago, Illinois, 5-10 November, 2006 (unpublished), p. 13353.

${ }^{9}$ C. Charles, B. Bonello, and F. Gannot, Ultrasonics 44, e1209 (2006).

${ }^{10}$ J. O. Vasseur, P. A. Deymier, B. Djafari-Rouhani, Y. Pennec, and A. C. Hladky-Hennion, Phys. Rev. B 77, 085415 (2008).

${ }^{11}$ Y. Pennec, B. Djafari-Rouhani, H. Larabi, J. O. Vasseur, and A.
C. Hladky-Hennion, Phys. Rev. B 78, 104105 (2008).

${ }^{12}$ T. T. Wu, Z. G. Huang, T.-C. Tsai, and T. C. Wu, Appl. Phys. Lett. 93, 111902 (2008).

${ }^{13}$ Y. Pennec, B. Djafari-Rouhani, H. Larabi, A. Akjouj, J. N. Gillet, J. O. Vasseur, and G. Thabet, Phys. Rev. B 80, 144302 (2009).

${ }^{14}$ T.-C. Wu, T-.T. Wu, and J.-C. Hsu, Phys. Rev. B 79, 104306 (2009).

${ }^{15}$ E. Yablonovitch, J. Opt. Soc. Am. B 10, 283 (1993).

${ }^{16}$ S. G. Johnson, S. Fan, P. R. Villeneuve, J. D. Joannopoulos, and L. A. Kolodziejski, Phys. Rev. B 60, 5751 (1999).

${ }^{17}$ S. Shi, C. Chen, and D. W. Prather, J. Opt. Soc. Am. A 21, 1769 (2004).

${ }^{18}$ V. V. Poborchii, T. Tada, and T. Kanayama, J. Appl. Phys. 91, 3299 (2002).

${ }^{19}$ Y. Zhao and D. Grishkowsky, Opt. Lett. 31, 1534 (2006).

${ }^{20}$ P. Lacharmoise, A. Fainstein, B. Jusserand, and V. Thierry-Mieg, Appl. Phys. Lett. 84, 3274 (2004).

${ }^{21}$ M. Maldovan and E. L. Thomas, Appl. Phys. Lett. 88, 251907 (2006).

${ }^{22}$ M. Maldovan and E. L. Thomas, Appl. Phys. B: Lasers Opt. 83, 595 (2006).

${ }^{23}$ S. Sadat-Saleh, S. Benchabane, F. I. Baida, M. P. Bernal, and V. Laude, J. Appl. Phys. 106, 074912 (2009).

${ }^{24}$ A. V. Akimov, Y. Tanaka, A. B. Pevtsov, S. F. Kaplan, V. G. Golubev, S. Tamura, D. R. Yakovlev, and M. Bayer, Phys. Rev. 
Lett. 101, 033902 (2008).

${ }^{25}$ A. H. Safavi-Naeini and O. Painter, Opt. Express 18, 14926 (2010).

${ }^{26}$ Y. Pennec, B. Djafari-Rouhani, E. H. El Boudouti, C. Li, Y. El Hassouani, J. O. Vasseur, N. Papanikolaou, S. Benchabane, V.
Laude, and A. Martinez, Opt. Express 18, 14301 (2010).

${ }^{27}$ S. Mohammadi, A. A. Eftekhar, A. Khelif, and A. Adibi, Opt. Express 18, 9164 (2010).

${ }^{28}$ J. O. Vasseur, P. A. Deymier, B. Chenni, B. Djafari-Rouhani, L. Dobrzynski, and D. Prevost, Phys. Rev. Lett. 86, 3012 (2001). 